07.3

\title{
Исследование радиофотонного принципа построения реактивного блокиратора радиоуправляемых взрывных устройств
}

\author{
() М.Е. Белкин, А.В. Алешин, Д.А. Фофранов, А.С. Сигов \\ МИРЭА - Российский технологический университет, Москва, Россия \\ E-mail: belkin@mirea.ru
}

Поступило в Редакцию 11 июня 2020 г.

В окончательной редакции 5 августа 2020 г.

Принято к публикации 5 августа 2020 г.

\begin{abstract}
Предложен и предварительно исследован новый подход к построению сверхширокополосного устройства блокирования радиоуправляемых взрывных устройств, в котором многоканальный блок обработки на основе набора узкополосных узлов приема радиосигналов с введением в каждый канал шумовой помехи заменен на одноканальный радиофотонный аналог с использованием для подавления канала передачи кода подрыва оптоэлектронного радиосигнального процессора на базе оптической рециркуляционной схемы. Описываются схема и результаты компьютерного моделирования оптоэлектронного процессора при передаче стандартных телекоммуникационных радиосигналов, подтверждающие возможность и определяющие основные условия блокирования радиоканала.
\end{abstract}

Ключевые слова: радиофотоника, блокиратор радиоуправляемых взрывных устройств, оптоэлектронный радиосигнальный процессор, компьютерное моделирование.

DOI: 10.21883/PJTF.2020.22.50305.18420

Наиболее ярким историческим примером современного развития радиоэлектронных систем блокирования радиоуправляемых взрывных устройств является многолетняя борьба вооруженных сил США с так называемыми самодельными взрывными устройствами (СВУ), что привело к созданию в стране самой мощной в мире промышленной отрасли [1]. Проблема противодействия террористическим атакам пусть и в меньшей степени, но также остро стоит в остальных странах мира [2], особенно в европейских странах, в которые вместе с беженцами из Северной Африки пришли и действуют члены исламских террористических группировок. Для оперативного решения проблемы в ряде промышленно развитых стран, включая Россию, созданы соответствующие органы и организации и налажен промышленный выпуск блокираторов радиоуправляемых взрывных устройств (БРВУ). Кратко охарактеризуем современное состояние и уровень их развития.

В настоящее время для дистанционного управления детонатором заложенного на объекте взрывчатого вещества различными террористическими группировками и индивидуальными террористами, как правило, используются самодельные взрывные радиоустройства (доработанные сотовые телефоны, переносные радиостанции), действующие в сверхширокополосном диапазоне 20-2700 MHz, охватывающем более семи октав. Кроме того, в связи с бурным развитием в мире мобильных телекоммуникационных сетей пятого поколения (5G NR) в ближайшее время ожидается расширение диапазона их работы до $6 \mathrm{GHz}[3]$, что необходимо учитывать при разработке средств противодействия нового поколения.

В общем современные БРВУ подразделяются по принципу работы на два типа [4]: активные, которые постоянно излучают так называемую заградительную помеху во всем диапазоне рабочих частот, и реактивные, которые сканируют радиоэфир и выдают так называемую прицельную помеху в заданной полосе частот, только если они обнаружат передачу радиосигнала в этой полосе. Первый тип более экономичный, однако его важным недостатком является создание помех не только подавляемому взрывному радиоустройству, но и всем устройствам сотовой связи в радиусе нескольких километров, поэтому они неприменимы в большом городе. Второй тип вследствие избирательных свойств получил более широкое распространение.

Структурная схема БРВУ с предложенным оптоэлектронным радиосигнальным процессором (ОЭРП) представлена на рис. 1. Как следует из рисунка, основными элементами схемы являются приемная антенна, малошумящий электронный усилитель 1, ОЭРП 2, электронный усилитель мощности 3 и передающая антенна. В состав ОЭРП входят полупроводниковый лазерный модуль 4 и фотодиодный модуль 5 , с помощью

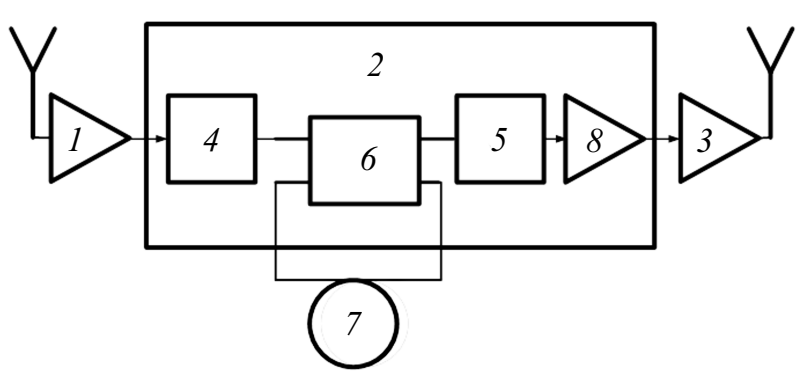

Рис. 1. Структурная схема предложенного БРВУ на базе ОЭРП. Пояснение в тексте. 
Основные исходные данные для моделирования

\begin{tabular}{|c|c|c|}
\hline \multicolumn{2}{|c|}{ Параметр } & Значение \\
\hline \multicolumn{2}{|c|}{$\begin{array}{l}\text { Длина псевдослучайной битовой последовательности } \\
\text { Диапазон частот радиосигнала } \\
\text { Формат модуляции радиосигнала } \\
\text { Максимальный символьный интервал } \\
\text { Отношение сигнала к шуму на входе ОЭРП }\end{array}$} & \begin{tabular}{c|c}
\multicolumn{3}{c}{$2^{15}-1$} \\
$0.02-6 \mathrm{GHz}$ \\
$\begin{array}{c}0 M \mathrm{sK} \\
4 \mu \mathrm{s}\end{array}$ & $\begin{array}{c}16-\mathrm{QAM} \\
100 \mu \mathrm{s}\end{array}$
\end{tabular} \\
\hline Полупроводниковый лазерный модуль & $\begin{array}{c}\text { Тип лазерного излучателя } \\
\text { Полоса модуляции } \\
\text { Рабочий ток } \\
\text { Средняя мощность } \\
\text { Оптическая несущая } \\
\text { Ширина линии излучения }\end{array}$ & $\begin{array}{c}\text { POC } \\
6 \mathrm{GHz} \\
50 \mathrm{~mA} \\
6 \mathrm{~mW} \\
1552.522 \mathrm{~nm} \\
1 \mathrm{MHz}\end{array}$ \\
\hline Фотодиодный модуль & $\begin{array}{c}\text { Чувствительность } \\
\text { Темновой ток } \\
\text { Полоса пропускания по уровню } 3 \mathrm{~dB}\end{array}$ & $\begin{array}{l}0.9 \mathrm{~A} / \mathrm{W} \\
100 \mathrm{nA} \\
10 \mathrm{GHz}\end{array}$ \\
\hline Предварительный усилитель & $\begin{array}{c}\text { Коэффициент усиления } \\
\text { Спектральная плотность шума }\end{array}$ & $\begin{array}{c}30-50 \mathrm{~dB} \\
20 \cdot 10^{-12} \mathrm{~A} / \mathrm{Hz}^{1 / 2}\end{array}$ \\
\hline Оптическая схема задержки & $\begin{array}{c}\text { Потери в } X \text {-разветвителе } \\
\text { Тип ОВ } \\
\text { Диапазон задержек в ОВ }\end{array}$ & $\begin{array}{c}3.5 \mathrm{~dB} \\
\mathrm{SMF}-28+ \\
1-125 \mu \mathrm{s}\end{array}$ \\
\hline
\end{tabular}

которых осуществляется прямой и обратный перенос принятого радиосигнала в оптический диапазон, расположенная между ними пассивная оптическая схема задержки (ОС3) рециркуляционного типа на базе оптического разветвителя $6 X$-типа и отрезка оптического волокна 7 определенной длины, с помощью которой осуществляется соответствующая обработка сигнала в оптическом диапазоне с целью создания интерференции с исходным радиосигналом так, чтобы ухудшить его качество настолько, чтобы после передачи в эфир он не мог быть распознан установленным на детонаторе взрывчатого вещества радиоприемным устройством. Для компенсации потерь при электрооптическом и оптикоэлектрическом преобразованиях в схеме ОЭРП после фотодиодного модуля введен предварительный электронный усилитель 8 . В принципе для создания большого числа копий также пригодна многоотводная ОС3. Однако из соображений компактности радиофотонного БРВУ был выбран рециркуляционный тип ОСЗ.

Проведенный сравнительный анализ позволил сделать следующие выводы относительно принципа построения и недостатков современных БРВУ.

I. Bce - как серийно выпускаемые, так и разрабатываемые - модели БРВУ построены на базе традиционного чисто радиоэлектронного подхода.

II. К общим принципиальным недостаткам существующих БРВУ можно отнести следующие:

1) необходимость частотного сканирования в указанном выше сверхширокополосном частотном диапазоне, что приводит к относительно невысокой скорости ре- акции устройства (от сотен микросекунд до единиц миллисекунд);

2) необходимость компьютерного управления для хранения и дополнительного введения в принятые сигналы маскирующей шумовой помехи из библиотеки оптимальных помех, что также уменьшает скорость реакции;

3) сложность схемы, что приводит к ухудшению массогабаритных характеристик и увеличению стоимости изделия (тем более что ОЭРП может быть выполнен в виде радиофотонной интегральной схемы, что приведет к еще более значительному уменьшению габаритов БРВУ).

Тем не менее необходимо подтвердить эффективность работы предложенного ОЭРП и определить технические параметры его ключевых элементов, неоптимальность которых может привести к ухудшению надежности закрытия канала связи и увеличению времени реакции. Потенциальными источниками, требующими оптимизации в схеме ОЭРП (рис. 1), являются соотношение между длительностью входного импульсного радиосигнала и временем задержки в ОС3, минимально допустимый коэффициент передачи ОЭРП, что будет исследовано далее путем компьютерного моделирования эффекта блокирования канала связи при подаче на его вход стандартных телекоммуникационных радиосигналов.

Параметрами расчета являются длительность входного радиоимпульса, которая соответствует числу символьных интервалов (СИ) передаваемого цифрового радиосигнала, время задержки в ОС3 за один проход $\tau$, число проходов $N$ и коэффициент передачи $A$ ОЭРП. Время 

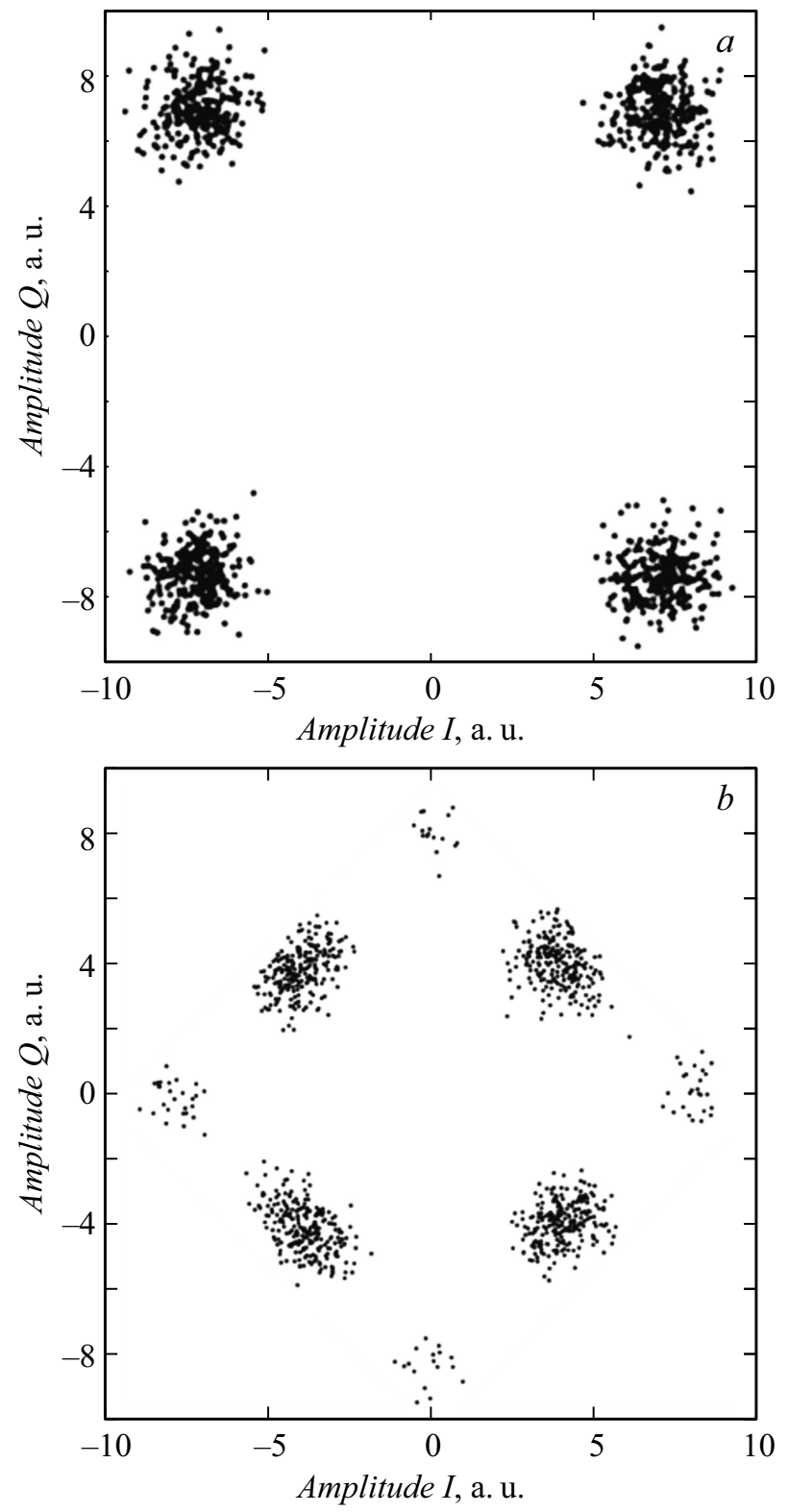

Pис. 2. Звездные диаграммы на выходе ОЭРП при передаче цифрового радиосигнала в формате GMSK. $a$ - открытый канал радиосвязи, $b-$ закрытый канал радиосвязи.

задержки $\tau$ определяется как [5]:

$$
\tau=L n_{f} / c
$$

где $L$ - длина оптического волокна в ОС $3, c-$ скорость света, $n_{f}-$ групповой показатель преломления оптического волокна $(\mathrm{OB})\left(n_{f}=1.47\right.$ для стандартного одномодового ОВ). Кроме того, коэффициент передачи $A$ зависит от потерь в элементах $4-7$, а также от коэффициента усиления элемента 8 схемы ОЭРП (рис. 1).

Для подтверждения эффективности функционирования предложенной схемы были разработаны компьютерные модели ОЭРП в универсальной системе автоматизи- рованного проектирования (САПР) Matlab и в специализированной оптоэлектронной CAПР OptiSystem. В таблице приведены основные исходные данные, которыми мы руководствовались при проведении модельных экспериментов. В частности, с целью практической направленности исследования выбраны два широко распространенных в современной сотовой связи формата цифровой модуляции обрабатываемого радиосигнала: гауссовская частотная модуляция с минимальным сдвигом GMSK (стандарт GSM ETSI TS 300 553) и 16-позиционная квадратурная амплитудная модуляция 16-QAM (стандарт 5G NR ETSI TS 136 104). Оценка закрытия канала радиосвязи проводилась качественно по звездной диаграмме (ЗД), в которой значения комплексной амплитуды радиосигнала $(I, Q)$ представляются в виде точек на двумерной точечной диаграмме на комплексной плоскости, и количественно по величине BER (bit error rate) либо EVM (error vector magnitude) [6]. Критериями надежного закрытия считались в соответствии с указанными выше стандартами следующие: $\mathrm{EVM} \geq 17.5 \%$ для GMSK, $\mathrm{EVM} \geq 12.5 \%$ для 16-QAM и $\mathrm{BER} \geq 0.1$ для обоих форматов модуляции.

Далее приводятся результаты модельных экспериментов. Для краткости их пояснения принята следующая терминология: случай отсутствия подавления радиосигнала СВУ будет называться открытым каналом радиосвязи, которому соответствует „неискаженная“ ЗД на выходе ОЭРП. И наоборот, случай подавления радиосигнала СВУ будет называться закрытым каналом радиосвязи, которому соответствует „искаженная“ ЗД на выходе ОЭРП.

Пример ЗД на выходе ОЭРП при передаче управляющего радиосигнала с GMSK представлен на рис. 2. В частности, на рис. 2, $a$ показана неискаженная ЗД при $\mathrm{BER}=10^{-8}$, обеспечивающем открытый канал радиосвязи, а на рис. $2, b-$ искаженная ЗД при $\mathrm{BER}=0.3$, полученная при следующих параметрах: $\tau=0.5 \mathrm{CИ}$, $N=2, A=20 \mathrm{~dB}$. Пример ЗД на выходе ОЭРП при передаче управляющего радиосигнала с 16 -QАМ представлен на рис. 3. В частности, на рис. 3, $a$ показана неискаженная ЗД при $\mathrm{EVM}=2.6 \%$, обеспечивающем открытый канал радиосвязи, а на рис. $3, b-$ искаженная ЗД при $\mathrm{EVM}=27 \%$, полученная при тех же параметрах расчета.

Как видно из рисунков, эффект закрытия канала радиосвязи приводит к существенному искажению звездной диаграммы, выражающемуся в уменьшении расстояния между двумя ближайшими точками созвездия, их смещении как по оси $I$, так и по оси $Q$, появлении ложных точек и т.д. В ходе моделирования также проведен анализ влияния числа проходов в ОСЗ $N$ на диапазон задержек $\tau$, в котором обеспечивается надежное закрытие канала радиосвязи, что обеспечивает универсальное применение предложенного подхода к построению БРВУ при использовании различных стандартов цифровой радиосвязи. В результате показано, 

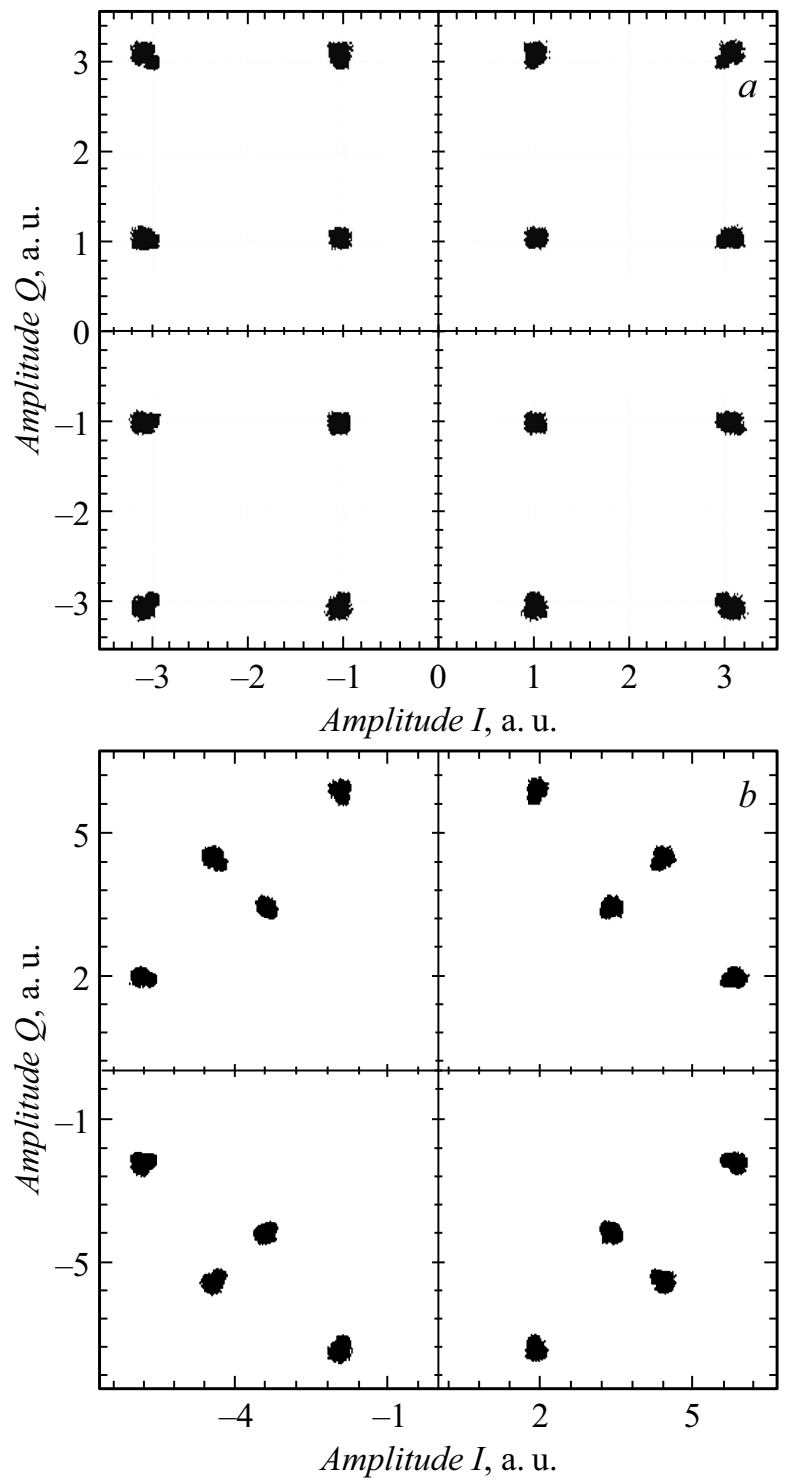

Рис. 3. Звездные диаграммы на выходе ОЭРП при передаче цифрового радиосигнала в формате 16-QAМ. $a$ - открытый канал радиосвязи, $b-$ закрытый канал радиосвязи.

что увеличение $N$ приводит к значительному расширению диапазона задержек. В частности, уже при $N=3$ диапазон значений $\tau$ по уровню $\mathrm{BER} \geq 0.1$ составляет от 0.3 до 3 СИ. Детальная проверка универсальности применения предложенного оптоэлектронного принципа построения БРВУ при передаче команды дистанционного подрыва в любых стандартах цифровой и аналоговой радиосвязи является темой наших дальнейших исследований.

\section{Финансирование работы}

Работа выполнена при поддержке Министерства науки и высшего образования России (шифр проекта RFMEFI60719X0319).

\section{Конфликт интересов}

Авторы заявляют, что у них нет конфликта интересов.

\section{Список литературы}

[1] Zoroya G. How the IED changed the U.S. military // USA Today. 2013. http://www.usatoday.com/story/news/nation/ 2013/12/18/ied-10-years-blast-wounds-amputations/3803017/

[2] Global terrorism index 2019. Measuring the impact of terrorism. Institute for Economics \& Peace, 2019. http://visionofhumanity.org/app/uploads/2019/11/GTI-2019web.pdf

[3] Mileusnić M., Petrović P., Pavić B., Marinković-Nedelicki V., Glišović J., Lebl A., Marjanović I. The radio jammer against remote controlled improvised explosive devices // Proc. of the 25th Telecommunications Forum (TELFOR). IEEE, 2017. P. 151-154. DOI: 10.1109/TELFOR.2017.8249309

[4] Mietzner J., Nickel P., Meusling A., Loos P., Bauch G. // IEEE Commun. Mag. 2012. V. 50. P. 38-46. DOI: 10.1109/MCOM.2012.6316774

[5] Urick V.J., McKinney J.D., Williams K.J. Fundamentals of microwave photonics. Hoboken, N.J.: Wiley, 2015. 496 p.

[6] Sklar B. Digital communications: fundamentals and applications. 2nd ed. London: Pearson, 2001. 1104 p. 